\title{
Plasma concentrations of lipoproteins and risk of lower-limb peripheral artery disease in people with type 2 diabetes: the SURDIAGENE study
}

\author{
Capucine Bertrand ${ }^{1} \cdot$ Pierre-Jean Saulnier ${ }^{2,3,4} \cdot$ Louis Potier $^{5,6,7}$ (D) $\cdot$ Mikaël Croyal ${ }^{8,9} \cdot$ Valentin Blanchard $^{9}$. \\ Elise Gand ${ }^{3}$. Stéphanie Ragot ${ }^{2,3,4}$ • Fabrice Schneider ${ }^{2,10}$ - Olivia Bocock ${ }^{1}$. Laurence Baillet-Blanco ${ }^{1}$. Gilberto Velho ${ }^{7}$. \\ Michel Marre ${ }^{6,7,11}$ (I) $\cdot$ Ronan Roussel ${ }^{5,6,7}$ (D) $\cdot$ Vincent Rigalleau $^{1,12,13} \cdot$ Samy Hadjadj $^{14}$ (I) $\cdot$ Kamel Mohammedi $^{1,11,15}$ (D) \\ on behalf of the SURDIAGENE Study Group
}

Received: 4 August 2020 / Accepted: 9 September 2020 / Published online: 6 January 2021

(C) Springer-Verlag GmbH Germany, part of Springer Nature 2021

\begin{abstract}
Aims/hypothesis The lipid profile has not been fully investigated in individuals with peripheral artery disease (PAD). We aimed to evaluate the relationship between plasma concentrations of lipoproteins and the prevalence of lower-limb PAD at baseline and its incidence during follow-up in people with type 2 diabetes.

Methods Plasma concentrations of total cholesterol, HDL-cholesterol, triacylglycerol and apolipoprotein (Apo) A-I, ApoA-II, ApoB-100 and Apo(a) were measured at baseline using colorimetric or MS methods in the SURDIAGENE cohort. Total cholesterol/HDL-cholesterol ratio, non-HDL-cholesterol and LDL-cholesterol were estimated using computation formulas. Logistic and Cox proportional hazard regression models were fitted to estimate OR or HR, with related 95\% CI, for baseline prevalence or incidence of major PAD (lower-limb amputation or requirement of revascularisation) during follow-up by increasing lipoprotein tertiles, after adjustment for key confounders.

Results Among 1468 participants (women 42\%, mean \pm SD age $65 \pm 11$ years, duration of diabetes $14 \pm 10$ years at baseline), $129(8.8 \%)$ had a baseline history of major PAD. Major PAD was less prevalent at baseline in the highest (vs lowest) tertile of HDL-cholesterol (OR 0.42 [95\% CI 0.26, 0.71], $p=0.001$ ) and ApoA-I (OR 0.39 [95\% CI 0.23, 0.67], $p=0.0007$ ), and more frequent in the highest tertile of total cholesterol/HDL-cholesterol ratio (OR 1.95 [95\% CI 1.18, 3.24], $p=0.01$ ). Among 1339
\end{abstract}

Capucine Bertrand and Pierre-Jean Saulnier are joint first authors. Samy Hadjadj and Kamel Mohammedi are joint senior authors.

Kamel Mohammedi

km.mmohammedi@gmail.com

1 Département d'Endocrinologie, Diabétologie, Nutrition, Hôpital Haut-Lévêque, Pessac, Bordeaux, France

2 UFR de Médecine et Pharmacie, Université de Poitiers, Poitiers, France

3 CHU de Poitiers, Centre d'Investigation Clinique, Poitiers, France

4 Inserm, CIC 1402, Poitiers, France

5 Assistance Publique - Hôpitaux de Paris, Bichat Hospital, DHU FIRE, Département d'Endocrinologie, Diabétologie, Nutrition, Paris, France

6 UFR de Médecine, Université de Paris, Paris, France

7 Centre de Recherche des Cordeliers, Inserm, Sorbonne Université, Université de Paris, Paris, France
8 INRA, CHU Nantes, UMR 1280, PhAN, IMAD, Nantes Université, Nantes, France

9 CRNH-O, Mass Spectrometry Core Facility, Nantes, France

10 Département de Chirurgie Vasculaire, CHU de Poitiers, Poitiers, France

11 CMC Ambroise Paré, Neuilly-sur-Seine, France

12 UFR de Médecine, Université de Bordeaux, Bordeaux, France

13 Centre de Recherche Inserm - Université de Bordeaux U1219 'Bordeaux Population Health', Bordeaux, France

14 Institut du Thorax, Inserm, CNRS, Université de Nantes, Nantes, France

15 Inserm U1034, Biologie des Maladies Cardiovasculaires, Bordeaux, France 


\section{Research in context}

\section{What is already known about this subject?}

- Dyslipidaemia affects $50 \%$ of individuals with type 2 diabetes and has been linked to both coronary and cerebrovascular atherosclerotic diseases

- Few studies have examined the relationship between dyslipidaemia and peripheral artery disease (PAD) in people with type 2 diabetes, with contrasting results

\section{What is the key question?}

- What is the profile of lipoproteins associated with major PAD in individuals with type 2 diabetes?

\section{What are the new findings?}

- Major PAD (lower-limb amputation or revascularisation) was less prevalent at baseline in the top (vs bottom) tertile of HDL-cholesterol and ApOA-I, and more frequent in the top tertile of total cholesterol/HDL-cholesterol ratio

- Among 1339 participants without a baseline history of PAD, the incidence of major PAD decreased in the top (vs bottom) tertile of HDL-cholesterol or ApoA-I and increased in the top tertile of total cholesterol/HDL-cholesterol ratio and non-HDL-cholesterol

- These associations were independent of putative confounders and mainly reliable after treating all-cause death or coronary events as competing risks

\section{How might this impact on clinical practice in the foreseeable future?}

- Individuals with type 2 diabetes and low plasma concentrations of HDL-cholesterol and ApoA-I or high total cholesterol/HDL-cholesterol ratio and non-HDL-cholesterol should be more strongly monitored to prevent the development of major adverse limb events

participants without a history of PAD at baseline, incident PAD occurred in $97(7.2 \%)$ during a median (25th-75th percentile) duration of follow-up of 7.1 (4.4-10.7) years, corresponding to 9685 person-years and an incidence rate of 9.8 (95\% CI 8.0, 12.0) per 1000 person-years. The risk of incident PAD was lower in the top (vs bottom) tertile of HDL-cholesterol (HR 0.54 [95\% CI $0.30,0.95], p=0.03$ ) or ApoA-I (HR 0.50 [95\% CI 0.28, 0.86], $p=0.01)$ and higher in the top tertile of total cholesterol/HDLcholesterol ratio (HR 2.81 [95\% CI 1.61, 5.04], $p=0.0002$ ) and non-HDL-cholesterol (HR 1.80 [95\% CI 1.06, 3.12], $p=0.03$ ). Conclusions/interpretation We reported independent associations between HDL-cholesterol, ApoA-I, total cholesterol/HDLcholesterol ratio or non-HDL-cholesterol and the prevalence or the incidence of major PAD in people with type 2 diabetes. Our findings provide a picture of lipoprotein profile in people with type 2 diabetes.

Keywords Apolipoproteins - Limb loss · Lipids · Lipoproteins · Lower-limb amputation · Peripheral arterial disease · Revascularisation - Type 2 diabetes

\section{Abbreviations}

\section{ACR}

Apo(a)

ApoA-I

ApoA-II

ApoB-100

$\mathrm{Lp}(\mathrm{a})$

PAD

SURDIAGENE
Albumin/creatinine ratio

Apolipoprotein (a)

Apolipoprotein A-I

Apolipoprotein A-II

Apolipoprotein B100

Lipoprotein (a)

Peripheral artery disease

SURVIe, DIAbete de type 2 et GENEtique

\section{Introduction}

Lower-limb peripheral artery disease (PAD) is an emerging public health burden with an endemic progression worldwide resulting from demographic expansion, population ageing and growing prevalence of type 2 diabetes and smoking habits [1, 2]. PAD is more prevalent in individuals with type 2 diabetes than in people without diabetes, with a poor prognosis leading to negative impacts on individual quality of life, healthcare 
systems and societies [3-5]. PAD is responsible for a dramatic increase in risk of non-traumatic lower-limb amputation, 5-12 times higher in individuals with diabetes than in those without a history of diabetes $[3,6]$. PAD is also associated with excess risk of CVD and non-CVD with a significant reduction in life expectancy $[5,7,8]$.

Dyslipidaemia affects about $50 \%$ of individuals with type 2 diabetes and is a major independent and modifiable risk factor for ischaemic CVD [9]. Atherogenic dyslipidaemia has been linked to both coronary and cerebrovascular atherosclerotic localisations [10, 11]. Despite considerable research on lipid metabolism and its impact in the development of CVD, only few reports dealt with PAD in individuals with diabetes. The specific lipoprotein components that contribute to PAD are not clearly established. In the present study, we investigated the profile of lipoproteins associated with the prevalence of major PAD at baseline and its incidence during follow-up in individuals with type 2 diabetes. Hence, we measured plasma concentrations of a range of lipid variables, including total cholesterol, HDL-cholesterol, LDL-cholesterol, non-HDLcholesterol, triacylglycerol, apolipoprotein A-I (ApoA-I), apolipoproten A-II (ApoA-II), apolipoprotein B100 (ApoB100 ) and lipoprotein (a) $[\mathrm{Lp}(\mathrm{a})]$ at baseline, and we investigated their relationships with the prevalence and the incidence of major PAD in a prospective cohort of individuals with type 2 diabetes.

\section{Methods}

Participants SURDIAGENE (SURvie, DIAbete de type 2 et GENEtique) is a French single-centre prospective cohort designed to investigate genetic and biochemical determinants of vascular complications among 1468 inpatient participants with type 2 diabetes diagnosed for at least 2 years [12]. The main exclusion criteria were the existence of a non-diabetic kidney disease and short followup duration $(<1$ year). Participants were recruited at the University Hospital of Poitiers, France, from 2002 to 2012, and were prospectively followed-up until death, or until 31 December 2015. The study protocol was approved by the Poitiers University Hospital Ethics Committee (CPP Ouest 3) and all participants gave written informed consent. The associations between lipoproteins and a baseline history of major PAD were tested in the whole cohort. Then, we tested the associations between lipoproteins and the incidence of major PAD in the incidence cohort, after exclusion of 129 individuals with a baseline history of PAD. The associations between lipoproteins and secondary endpoints (see below) were tested in participants without a baseline history of lower-limb amputation or revascularisation procedure as appropriate (electronic supplementary material [ESM] Fig. 1).
Assessments of plasma concentrations of lipoproteins Plasma concentrations of total cholesterol, HDL-cholesterol and triacylglycerol were determined centrally at baseline in the fasting state using a colorimetric method, running on an automated analyser (Kone Optima; Thermo Clinical Labsystems, Vantaa, Finland). The total cholesterol/HDLcholesterol ratio was calculated. Plasma concentrations of LDL-cholesterol were estimated using the Friedewald formula in 1409 participants with triacylglycerol $<4.5 \mathrm{mmol} / 1$. Non-HDL-cholesterol was calculated as total cholesterol value minus HDL-cholesterol. ApoA-I, ApoAII, ApoB-100 and apolipoprotein (a) [Apo(a)] were quantified in plasma samples $(40 \mu \mathrm{l})$ using a validated multiplexed assay involving trypsin proteolysis and the subsequent analysis of proteotypic peptides by LC-MS/ MS [13, 14]. The intra- and inter-assay imprecisions of the analytical method were assessed throughout experiments and were below $6.4 \%$ for all targeted apolipoproteins. Since $\mathrm{Lp}(\mathrm{a})$ consists of a single apolipoprotein [Apo(a)] bound to the ApoB-100 moiety of an LDL-like particle, the molar concentrations of Apo(a) were assumed to be equivalent to those of $\mathrm{Lp}(\mathrm{a})$. $\mathrm{Lp}$ (a) concentrations in units of $\mathrm{nmol} / \mathrm{l}$ were then converted to units of $\mathrm{mg} / \mathrm{l}$ using the following formula: $\mathrm{Lp}(\mathrm{a})(\mathrm{nmol} / \mathrm{l})=0.218 \times \mathrm{Lp}(\mathrm{a})(\mathrm{mg} /$ 1) -3.83 [15]. Hence, results are presented in this work as $\mathrm{Lp}$ (a) expressed in $\mathrm{mg} / \mathrm{l}$.

Definition of clinical conditions at baseline The history of tobacco smoking was defined as never, former or current smokers. Diabetic retinopathy was staged as absent, nonproliferative or proliferative. The history of macrovascular disease was defined as the presence of at least one of the following conditions: myocardial infarction; stable angina; stroke; transient ischaemic attack; or coronary or carotid arterial revascularisation. The prevalence of lower-limb PAD was defined as the history of minor (at least one toe or transmetatarsal) or major (transtibial or transfemoral) amputation, or revascularisation at baseline.

Definition of endpoints during follow-up The primary endpoint, incident major PAD, was defined as the first occurrence either of lower-limb amputation (transmetatarsal, transtibial or transfemoral) or the requirement of a lowerlimb revascularisation procedure (angioplasty or surgery) during follow-up. Revascularisation and lower-limb amputation were considered individually as secondary endpoints.

Adjudication procedure Outcomes were determined from participants' medical records and interviews with their general practitioners every second year from 2007 . The hospitalisation records and all other relevant supporting documents were used to adjudicate clinical outcomes. Each endpoint was centrally reviewed by an independent adjudication committee. 
Causes of lower-limb amputation We have examined participants' medical files to determine the potential causes of lowerlimb amputation at baseline (for prevalent amputation) and at the endpoint time (for incident amputation): neuropathy (as reported by the investigator); PAD (abolition of peripheral pulses, intermittent claudication, lower-limb artery stenosis $>50 \%$ with haemodynamic effects in ultrasound examination); and/or foot infection (skin, soft tissue, bone or joint).

Statistical analyses Categorical variables are expressed as the number of participants with corresponding percentage. Continuous variables are expressed as mean \pm SD or median (25th-75th percentile) for those with skewed distribution. Comparisons of characteristics of participants at baseline were performed using $\chi^{2}$, ANOVA or Wilcoxon tests. The correlation between different lipoproteins were assessed using Pearson or Spearman's rank test. Plasma concentrations of lipoproteins were categorised into three equal increasing tertiles: first (T1, lowest tertile); second (T2, middle tertile); and third (T3, highest tertile). Missing data were rare (Table 1) and were removed from all analyses that included the covariate.

Logistic regression models were used to test the associations between lipoproteins and the prevalence of major PAD at baseline, expressed as OR with related $95 \%$ CI for T2 vs T1 and T3 vs T1. Analyses were adjusted for every potential confounding variable that was nominally associated $(p<0.10)$ with the prevalence of major PAD at baseline in the univariate comparisons: sex; age; duration of diabetes; BMI; systolic and diastolic $\mathrm{BP}$; $\mathrm{HbA}_{1 \mathrm{c}}$; urinary albumin/ creatinine ratio (ACR, using a natural log transformation); eGFR (estimated using the Chronic Kidney DiseaseEpidemiology Collaboration equation); history of tobacco smoking (never, former, current); history of diabetic retinopathy; history of macrovascular disease; and history of medication use (antihypertensive, antiplatelet or anticoagulant drugs, statins, fibrates or metformin).

Restricted cubic splines (10th, 25th, 75th and 90th percentiles as knots and the median as reference) analyses were plotted to check for the linearity in the relationship between plasma concentrations of lipoproteins at baseline and the risk of incident PAD during follow-up.

Kaplan-Meier curves were used to plot the incidence of endpoints according to tertiles of lipoproteins at baseline and compared using logrank test. Cox proportional hazards regression models were computed to calculate HRs, with related 95\% CIs, for endpoints during follow-up by tertiles of lipoproteins at baseline (T2 vs T1 and T3 vs T1). Lipid variables with a linear PAD relationship were also tested as continuous variables (HR for the primary endpoint by each single SD increase). Analyses were adjusted for age plus every potential confounding variable that was nominally associated $(p<0.10)$ with the incidence of major PAD during follow-up in the univariate comparisons: sex; duration of diabetes; BMI; systolic BP; ACR; eGFR; history of tobacco smoking (never, former, current); diabetic retinopathy; and use of antihypertensive, statin, metformin and insulin therapies. We tested interaction between relevant lipid variables in their association with the primary endpoint by including them and their product within the Cox model. We also tested interaction between lipoproteins and the use of statins in PAD association. The proportional hazards assumption was checked using the Schoenfeld residuals method (all $p$ values $>0.05$ ).

As sensitivity analyses, we estimated the risk of the primary endpoint by plasma concentrations of lipoproteins after treating all-cause death or coronary events (myocardial infarction or requirement of coronary revascularisation, whichever came first) as competing risk using the Fine and Gray method.

Statistical analysis was performed using JMP software, version 14.0 (SAS Institute, Cary, NC, USA; www.sas.com) and Stata software version 15.1 (StataCorp, TX, USA; http:// www.stata.com).

\section{Results}

Characteristics of participants at baseline Among 1468 patients enrolled in SURDIAGENE, $42 \%$ were women and $10 \%$ were current smokers at baseline. The mean \pm SD age and duration of diabetes were $65 \pm 11$ years and $14 \pm 10$ years, respectively. The mean \pm SD plasma concentrations of lipids and lipoproteins were as follows: total cholesterol $4.8 \pm$ $1.2 \mathrm{mmol} / \mathrm{l}$; HDL-cholesterol $1.2 \pm 0.4 \mathrm{mmol} / \mathrm{l}$; non-HDLcholesterol $3.6 \pm 1.2 \mathrm{mmol} / \mathrm{l}$; LDL-cholesterol $2.7 \pm$ $1.0 \mathrm{mmol} / \mathrm{l}$; ApoA-I $1.3 \pm 0.3 \mathrm{~g} / \mathrm{l}$; ApoA-II $0.31 \pm 0.11 \mathrm{~g} / \mathrm{l}$; and ApoB-100 $0.81 \pm 0.29 \mathrm{~g} / 1$. The median $(25 \mathrm{th}-75$ th percentiles) of total cholesterol/HDL-cholesterol ratio, triacylglycerol and Lp(a) were 4.0 (3.1-5.2), 1.5 (1.1-2.3) mmol/1 and 74 (19-203) mg/l, respectively. Plasma concentrations of lipoproteins in different corresponding tertiles are presented in ESM Table 1, and the pairwise correlations are displayed in ESM Table 2.

Prevalence of major PAD by plasma concentrations of lipoproteins at baseline A history of major PAD was reported at baseline in $129(8.8 \%)$ patients. Table 1 shows the characteristics of participants by the baseline prevalence of major PAD. The mean \pm SD plasma concentrations of HDL-cholesterol $(1.1 \pm 0.3$ vs $1.2 \pm 0.4 \mathrm{mmol} / \mathrm{l}, p=0.003)$, ApoA-I $(1.2 \pm 0.2$ vs $1.3 \pm 0.3 \mathrm{~g} / 1, p<0.0001)$ and ApoA-II $(0.28 \pm 0.10$ vs 0.32 $\pm 0.11 \mathrm{~g} / \mathrm{l}, p=0.0002)$ were significantly lower in participants who had a history of major PAD at baseline compared with those who did not (Table 1). PAD at baseline was less prevalent in the highest compared with the lowest tertile of HDLcholesterol, Apo-A1 and Apo-A2, and more frequent in the highest tertile of total cholesterol/HDL-cholesterol ratio 
Table 1 Characteristics of participants at baseline according to the prevalence and the incidence of major PAD

Characteristic
Prevalence of major PAD at baseline Incidence of major PAD during follow-up ${ }^{\mathrm{a}}$

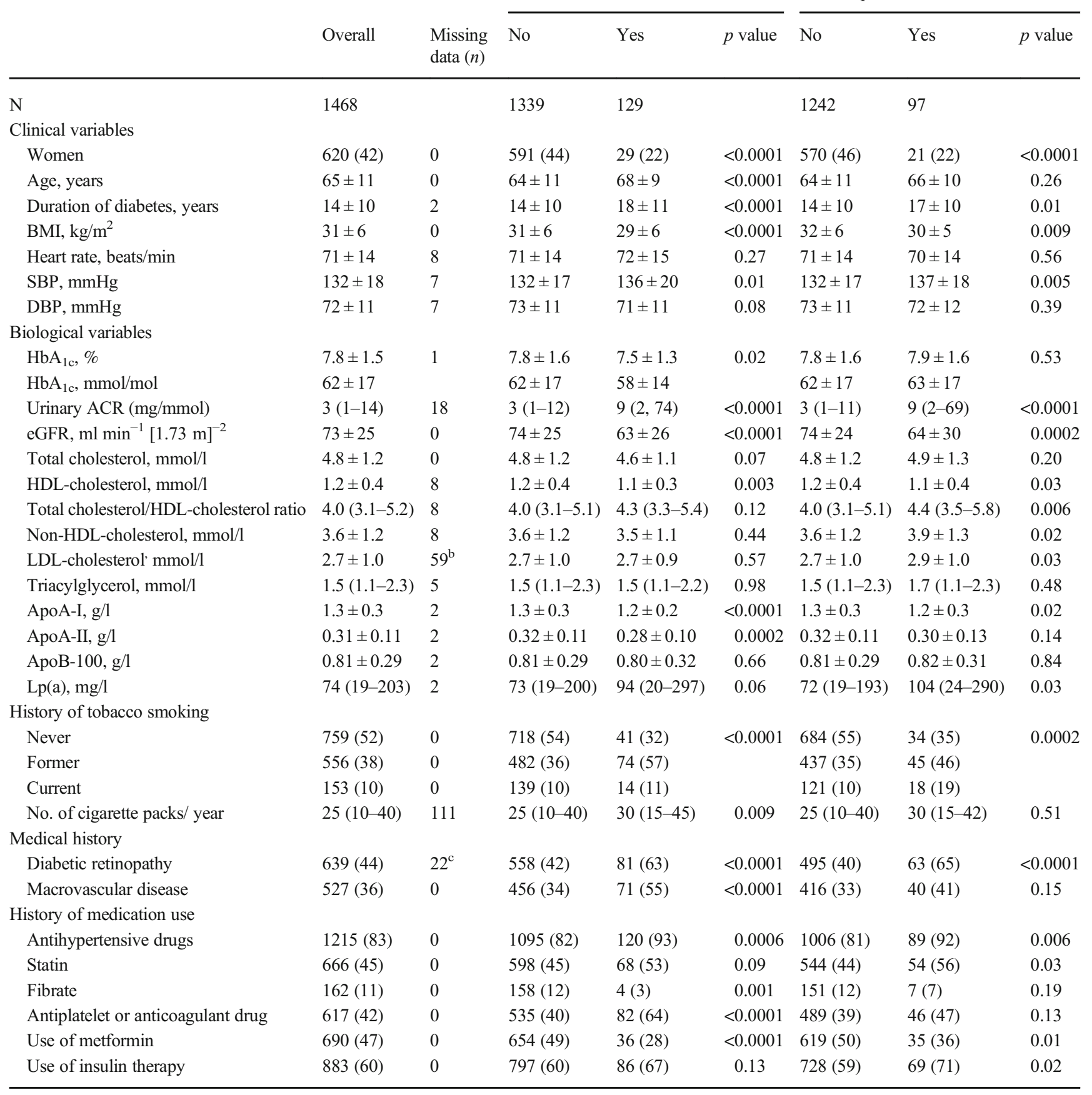

Data are presented as $n(\%)$ or mean $\pm \mathrm{SD}$, or as median (25th-75th percentiles) for variables with skewed distribution (urinary albumin to creatinine ratio, triacylglycerol, $\mathrm{Lp}(\mathrm{a})$, total cholesterol/HDL-cholesterol ratio and number of cigarette packs per year)

${ }^{a}$ Analyses performed in participants without a history of major PAD at baseline

${ }^{\mathrm{b}}$ Includes 51 participants who were excluded from the estimation (using the Friedewald formula) because plasma concentrations of triacylglycerol were high ( $>4.5 \mathrm{mmol} / \mathrm{l})$

${ }^{\mathrm{c}}$ Includes missing data and undetermined retinopathy status

Comparisons of qualitative and quantitative variables were performed using $\chi^{2}$ and ANOVA tests, respectively. Wilcoxon test was used for comparisons of variables with skewed distribution. $p<0.05$ was considered as significant

DBP, diastolic BP; SBP, systolic BP 
(Table 2; T3 vs T1). Logistic regression models confirmed these associations after adjustment for confounding variables (Table 2).

Prevalence of lower-limb amputation and revascularisation by plasma concentrations of lipoproteins at baseline A history of lower-limb amputation (74\% minor and $26 \%$ major) was reported at baseline in $73(5.0 \%)$ participants. They all had evidence of PAD (at least one of the following: abolition of peripheral pulses $62 \%$, intermittent claudication $42 \%$, lowerlimb artery stenosis $>50 \%$ with haemodynamic effects $59 \%$ ). Peripheral diabetic neuropathy and foot infection were also reported at baseline in $59 \%$ and $68 \%$ of participants with a history of amputation. A history of lower-limb revascularisation procedures was reported at baseline in 73 (5.0\%) participants. The highest tertiles of HDL-cholesterol and ApoA-I, compared with the respective lowest tertiles, were significantly associated with lower prevalence of lower-limb amputation and revascularisation at baseline (ESM Table 3).

Incidence of major PAD during follow-up by plasma concentrations of lipoproteins at baseline Among 1339 participants without a history of PAD at baseline, incident PAD occurred in $97(7.2 \%)$ during a median (25th-75th percentile) duration of follow-up of 7.1 (4.4-10.7) years, corresponding to 9685 person-years and an incidence rate of 9.8 (95\% CI 8.0, 12.0) per 1000 person-years. Characteristics of participants at baseline by incident PAD during follow-up are presented in Table 1.

Plasma concentrations of HDL-cholesterol and ApoA-I were significantly lower, while LDL-cholesterol, non-HDLcholesterol and Lp(a) were higher in participants who experienced a major PAD during follow-up compared with participants who had not (Table 1). The total cholesterol/HDLcholesterol ratio was also higher in participants who experienced major PAD during follow-up. The relationships between plasma concentrations of each lipid biomarker and the risk of major PAD during follow-up were not log-linear, except for ApoA-I (ESM Fig. 2).

The Kaplan-Meier estimate of 10 year cumulative incidence (95\% CI) of major PAD was significantly lower for participants in the highest (T3) vs lowest tertile (T1) of HDL-cholesterol (T1, 11.4 [8.0, 15.8]\%; T2, 10.8 [7.6, 15.3]\%; T3, 6.9 [4.4, $10.6] \%)$ and ApoA-I (T1, $13.1[9.0,18.1] \%$; T2, 10.1 [7.0, $14.3] \%$; T3, 6.4 [4.1, 10.0]\%) (Table 2, Fig. 1b,g). It was also reduced in the middle vs the lowest ApoA-II tertile (T1, 12.8 $[9.2,17.4] \%$; T2, $7.6[4.8,11.7] \%$; T3, $9.0[6.1,13.0] \%)$ and increased in the highest vs lowest tertile of total cholesterol/ HDL-cholesterol ratio (T1, $6.4[4.0,10.1] \%$; T2, 9.1 [6.2, $13.1] \%$; T3, 13.3 [9.2, 17.9]\%) (Table 2, Fig. 1c,h). HDLcholesterol, total cholesterol/HDL-cholesterol ratio and ApoA-I (but not ApoA-II) remained significantly associated with the risk of major PAD after adjusting for key confounders
(Table 2). These associations remained significant after considering all-cause death or coronary events as competing risks (ESM Table 4). Each single SD increase in ApoA-I was significantly associated with a reduced risk of major PAD (HR 0.76 [95\% CI 0.60, 0.96], $p=0.02$ ).

The highest tertile of non-HDL-cholesterol was also significantly associated with increased risk of major PAD after adjusting for confounders. However, this association did not persist after treating all-cause death or coronary events as competing risk (ESM Table 4). No other significant association was observed between lipids and major PAD (Table 2).

We observed a significant interaction between total cholesterol/HDL-cholesterol ratio and non-HDL-cholesterol in their association with the risk of incident PAD ( $p$ for interaction $=0.01)$. No other significant interaction was observed between HDL-cholesterol, total cholesterol/HDL-cholesterol ratio, non-HDL-cholesterol or ApoA-I in their association with the risk of major PAD ( $p$ values $>0.05$ ). In addition, no significant interaction was observed between HDL-cholesterol ( $p=$ $0.49)$, total cholesterol/HDL-cholesterol ratio $(p=0.86)$, nonHDL-cholesterol $(p=0.06)$ or ApoA-I $(p=0.16)$ with the use of statins in their association with major PAD.

Risks of incident lower-limb amputation and revascularisation by plasma concentrations of lipoproteins at baseline Lower-limb amputation (45\% minor and 55\% major) occurred during follow-up in 55 (3.9\%) participants without a baseline history of limb loss. Its incidence rate was 5.2 (95\% CI 4.0, 6.8) per 1000 person-years. Every participant who experienced limb loss during follow-up showed evidence of PAD at the time of outcomes (at least one of the following: abolition of peripheral pulses $76 \%$, intermittent claudication $56 \%$, lower-limb artery stenosis $>50 \%$ with haemodynamic effects 95\%). Peripheral diabetic neuropathy and foot infection were reported in $76 \%$ and $51 \%$ participants, respectively, among amputees at the time of endpoint. Requirement of revascularisation occurred during follow-up in $78(5.6 \%)$ participants without a history of this procedure at baseline. Its incidence rate was 7.7 (95\% CI 6.2, 9.7) per 1000 person-years. The risks of incident amputation and requirement of revascularisation, considered separately as secondary endpoints, were lower in the top tertiles of HDL-cholesterol and ApoA-I, and higher in the top tertile of total cholesterol/ HDL-cholesterol ratio, compared with the bottom tertiles (Table 3).

\section{Discussion}

In the present study, we investigated the relationship between lipid variables and the prevalence of PAD at baseline and its incidence during follow-up in individuals with type 2 diabetes. We observed lower prevalent and incident PAD in participants 
Table 2 Prevalence and incidence of PAD by tertiles of plasma lipoprotein concentration at baseline

\begin{tabular}{|c|c|c|c|c|c|c|c|c|c|}
\hline \multirow[t]{2}{*}{ Variable } & \multicolumn{4}{|c|}{ Prevalent major PAD at baseline ${ }^{a}$} & \multicolumn{5}{|c|}{ Incident major PAD during follow-up ${ }^{b}$} \\
\hline & No, $n$ & Yes, $n(\%)$ & OR $(95 \% \mathrm{CI})$ & $p$ value & No, $n$ & Yes, $n(\%)$ & $\begin{array}{l}10 \text { year cumulative } \\
\text { incidence, } \%(95 \% \mathrm{CI})\end{array}$ & HR $(95 \% \mathrm{CI})$ & $p$ value \\
\hline \multicolumn{10}{|l|}{ Total cholesterol } \\
\hline First tertile & 432 & $57(11.7)$ & Reference & & 415 & $31(7.0)$ & $10.2(6.9,14.9)$ & Reference & \\
\hline Second tertile & 449 & $40(8.2)$ & $0.93(0.58,1.49)$ & 0.76 & 420 & $26(5.8)$ & $7.0(4.5,10.7)$ & $1.01(0.58,1.73)$ & 0.98 \\
\hline Third tertile & 458 & $32(6.5)$ & $0.68(0.40,1.14)$ & 0.14 & 407 & $40(9.0)$ & $11.8(8.5,16.0)$ & $1.34(0.81,2.25)$ & 0.25 \\
\hline \multicolumn{10}{|l|}{ HDL-cholesterol } \\
\hline First tertile & 428 & $58(11.9)$ & Reference & & 406 & $37(8.4)$ & $11.4(8.0,15.8)$ & Reference & \\
\hline Second tertile & 447 & $40(8.2)$ & $0.63(0.40,1.00)$ & 0.05 & 408 & $36(8.1)$ & $10.8(7.6,15.3)$ & $1.00(0.62,1.62)$ & 0.98 \\
\hline Third tertile & 456 & $31(6.4)$ & $0.42(0.26,0.71)$ & 0.001 & 422 & $22(4.9)$ & $6.9(4.4,10.6)$ & $0.54(0.30,0.95)$ & 0.03 \\
\hline \multicolumn{10}{|c|}{ Total cholesterol/HDL-cholesterol ratio } \\
\hline First tertile & 448 & $38(7.8)$ & Reference & & 423 & $20(4.5)$ & $6.4(4.0,10.1)$ & Reference & \\
\hline Second tertile & 447 & $40(8.2)$ & $1.32(0.80,2.18)$ & 0.28 & 411 & $33(7.4)$ & $9.1(6.2,13.1)$ & $1.83(1.04,3.29)$ & 0.04 \\
\hline Third tertile & 436 & $51(10.5)$ & $1.95(1.18,3.24)$ & 0.01 & 402 & $42(9.5)$ & $13.3(9.2,17.9)$ & $2.81(1.61,5.04)$ & 0.0002 \\
\hline \multicolumn{10}{|c|}{ Non-HDL-cholesterol } \\
\hline First tertile & 436 & $50(10.3)$ & Reference & & 419 & $25(5.6)$ & $8.4(5.4,12.8)$ & Reference & \\
\hline Second tertile & 446 & $41(8.4)$ & $0.99(0.61,1.58)$ & 0.95 & 414 & $30(6.8)$ & $8.0(5.3,11.7)$ & $1.30(0.75,2.26)$ & 0.35 \\
\hline Third tertile & 449 & $38(7.8)$ & $1.04(0.63,1.74)$ & 0.86 & 403 & $40(9.0)$ & $12.2(8.8,16.5)$ & $1.80(1.06,3.12)$ & 0.03 \\
\hline \multicolumn{10}{|l|}{ LDL-cholesterol } \\
\hline First tertile & 418 & $51(10.9)$ & Reference & & 404 & $24(5.6)$ & $7.8(5.1,11.8)$ & Reference & \\
\hline Second tertile & 437 & $33(7.0)$ & $0.81(0.49,1.34)$ & 0.40 & 398 & $31(7.2)$ & $9.0(6.0,13.1)$ & $1.41(0.81,2.48)$ & 0.22 \\
\hline Third tertile & 431 & $39(8.3)$ & $0.97(0.58,1.61)$ & 0.89 & 392 & $37(8.6)$ & $11.2(8.0,15.5)$ & $1.71(0.99,3.00)$ & 0.05 \\
\hline \multicolumn{10}{|l|}{ Triacylglycerol } \\
\hline First tertile & 448 & $41(8.4)$ & Reference & & 415 & $29(6.5)$ & $7.6(5.0,11.3)$ & Reference & \\
\hline Second tertile & 444 & $44(9.0)$ & $1.23(0.76,1.99)$ & 0.39 & 413 & $32(7.2)$ & $11.0(7.0,15.7)$ & $1.22(0.73,2.04)$ & 0.45 \\
\hline Third tertile & 444 & $44(9.0)$ & $1.57(0.95,2.59)$ & 0.07 & 411 & $34(7.6)$ & $10.1(7.1,14.1)$ & $1.35(0.81,2.27)$ & 0.25 \\
\hline \multicolumn{10}{|l|}{ ApoA-I } \\
\hline First tertile & 431 & $57(11.7)$ & Reference & & 404 & $41(9.2)$ & $13.1(9.0,18.1)$ & Reference & \\
\hline Second tertile & 441 & $48(9.8)$ & $0.90(0.58,1.40)$ & 0.64 & 412 & $34(7.6)$ & $10.1(7.0,14.3)$ & $0.82(0.51,1.31)$ & 0.42 \\
\hline Third tertile & 465 & $24(4.9)$ & $0.39(0.23,0.67)$ & 0.0007 & 425 & $21(4.7)$ & $6.4(4.1,10.0)$ & $0.50(0.28,0.86)$ & 0.01 \\
\hline \multicolumn{10}{|l|}{ ApoA-II } \\
\hline First tertile & 426 & $62(12.7)$ & Reference & & 403 & $42(9.4)$ & $12.8(9.2,17.4)$ & Reference & \\
\hline Second tertile & 451 & $38(7.8)$ & $0.71(0.45,1.13)$ & 0.15 & 422 & $24(5.4)$ & $7.6(4.8,11.7)$ & $0.61(0.36,1.02)$ & 0.06 \\
\hline Third tertile & 460 & $29(5.9)$ & $0.59(0.36,0.98)$ & 0.04 & 416 & $30(6.7)$ & $9.0(6.1,13.0)$ & $0.90(0.55,1.48)$ & 0.69 \\
\hline \multicolumn{10}{|l|}{ ApoB-100 } \\
\hline First tertile & 440 & $48(9.8)$ & Reference & & 409 & $36(8.1)$ & $11.1(7.8,15.5)$ & Reference & \\
\hline Second tertile & 450 & $39(8.0)$ & $0.99(0.61,1.59)$ & 0.97 & 419 & $27(6.1)$ & $8.4(5.5,12.6)$ & $0.87(0.52,1.44)$ & 0.58 \\
\hline Third tertile & 447 & $42(8.6)$ & $1.35(0.84,2.19)$ & 0.22 & 413 & $33(7.4)$ & $9.7(6.8,13.8)$ & $1.21(0.74,1.98)$ & 0.43 \\
\hline \multicolumn{10}{|l|}{$\mathrm{Lp}(\mathrm{a})$} \\
\hline First tertile & 451 & $37(7.6)$ & Reference & & 419 & $27(6.1)$ & $8.7(5.7,12.9)$ & Reference & \\
\hline Second tertile & 450 & $39(8.0)$ & $1.05(0.63,1.73)$ & 0.85 & 418 & $28(6.3)$ & $9.0(6.0,13.1)$ & $0.79(0.46,1.37)$ & 0.40 \\
\hline Third tertile & 436 & $53(10.8)$ & $1.24(0.77,2.00)$ & 0.37 & 404 & $41(9.2)$ & $11.4(8.2,15.7)$ & $1.05(0.64,1.75)$ & 0.85 \\
\hline
\end{tabular}

${ }^{\text {a }}$ Associations between lipoproteins and baseline prevalence of major PAD were tested in the whole cohort using logistic regression models, adjusting for sex, age, duration of diabetes, BMI, systolic and diastolic $\mathrm{BP}, \mathrm{HbA}_{1 \mathrm{c}}$, urinary ACR (using a natural log transformation), eGFR, history of tobacco smoking (never, former, current), history of diabetic retinopathy or macrovascular disease and use of antihypertensive treatment, statin, fibrate, antiplatelet or anticoagulant drug and metformin

${ }^{\mathrm{b}}$ Associations between lipoproteins and incident PAD were tested in participants without a baseline history of PAD. The 10 year cumulative incidences, with associated 95\% CIs, were estimated using the Kaplan-Meier survival analyses. HR, with associated 95\% CIs, were computed using Cox proportional hazards regression models adjusting for age, sex, duration of diabetes, BMI, systolic BP, urinary ACR, eGFR, history of tobacco smoking (never, former, current), history of diabetic retinopathy and use of antihypertensive treatment, statin, metformin and insulin therapy

$p<0.05$ was significant 

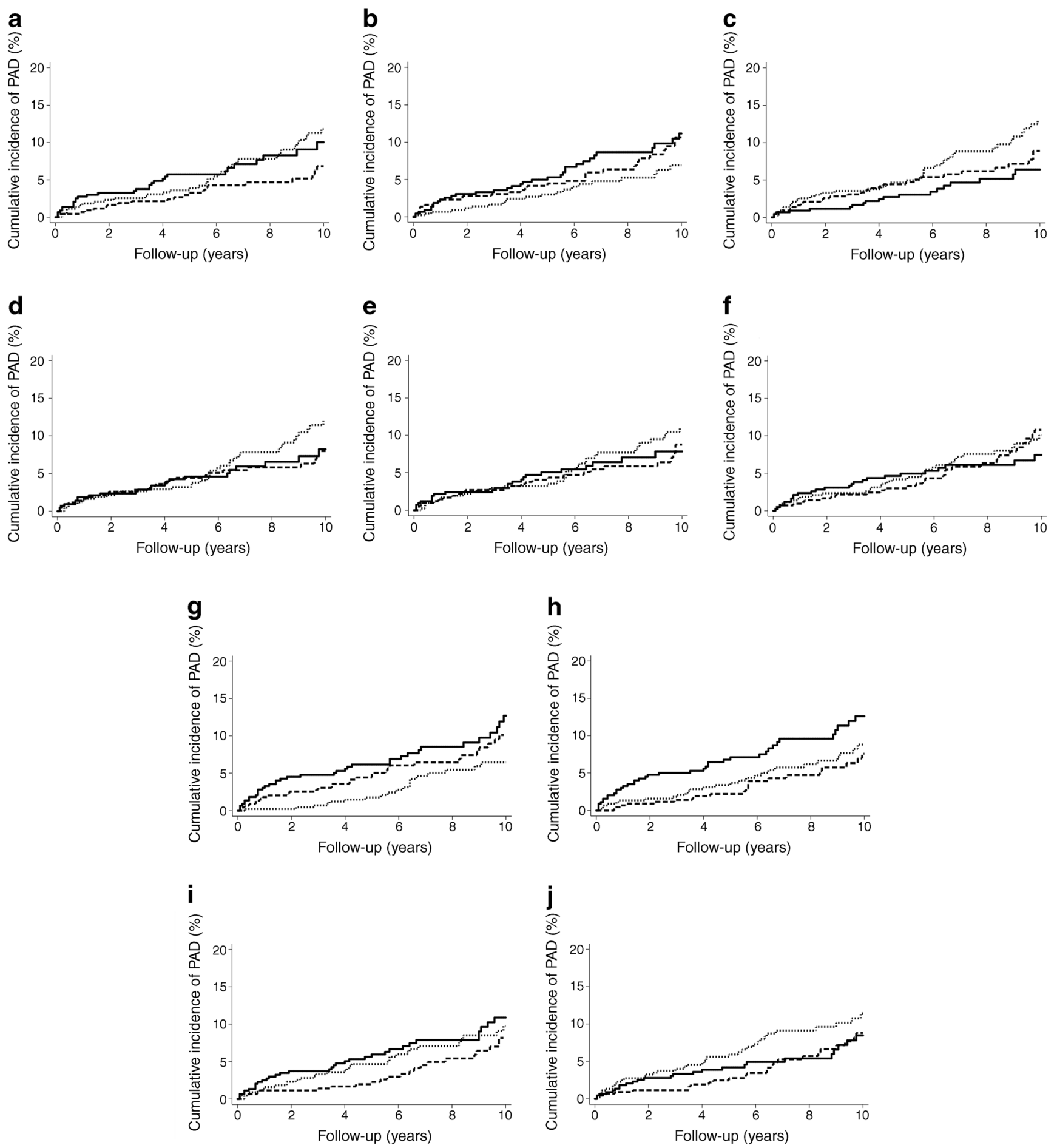

Fig. 1 Cumulative incidence of major PAD during follow-up according to the first (solid line), second (dashed line) and third tertiles (dotted line) of plasma concentrations of total cholesterol (a, $p=0.26)$, HDL-cholesterol $(\mathbf{b}, p=0.05)$, total cholesterol/HDL-cholesterol ratio $(\mathbf{c}, p=0.03)$, nonHDL-cholesterol (d, $p=0.41)$, LDL-cholesterol (e, $p=0.68)$,

triacylglycerol (f, $p=0.96)$, ApoA-I (g, $p=0.009)$, ApoA-II (h, $p=0.02)$, ApoB-100 (i, $p=0.43)$ and $\operatorname{Lp}(\mathrm{a})(\mathbf{j}, p=0.13)$. $p$ values provided from logrank test comparing the three tertiles for each lipid variable. Analyses performed in 1339 participants without a history of PAD at baseline

in the highest, compared with those in the lowest, tertiles of HDL-cholesterol and ApoA-I. In addition, the prevalence of major PAD increased in the upper tertiles of total cholesterol/ HDL-cholesterol ratio, compared with the lowest tertiles, and 
Table 3 Incidences of lower-limb amputation and revascularisation during follow-up by plasma concentrations of lipoproteins at baseline

\begin{tabular}{|c|c|c|c|c|c|c|c|c|c|c|}
\hline \multirow[t]{2}{*}{ Variable } & \multicolumn{5}{|c|}{ Lower-limb amputation during follow-up ${ }^{\mathrm{a}}$} & \multicolumn{5}{|c|}{ Lower-limb arterial revascularisation during follow-up ${ }^{\mathrm{b}}$} \\
\hline & No, $n$ & Yes, $n(\%)$ & $\begin{array}{l}10 \text { year } \\
\text { cumulative } \\
\text { incidence, } \\
\%(95 \% \mathrm{CI})^{\mathrm{c}}\end{array}$ & $\begin{array}{l}\text { HR } \\
(95 \% \mathrm{CI})^{\mathrm{d}}\end{array}$ & $p$ value & No, $n$ & Yes, $n(\%)$ & $\begin{array}{l}10 \text { year } \\
\text { cumulative } \\
\text { incidence, } \\
\%(95 \% \mathrm{CI})^{\mathrm{c}}\end{array}$ & $\begin{array}{l}\mathrm{HR} \\
(95 \% \mathrm{CI})^{\mathrm{d}}\end{array}$ & $p$ value \\
\hline \multicolumn{11}{|l|}{ Total cholesterol } \\
\hline First tertile & 446 & $19(4.1)$ & $5.9(3.5,9.7)$ & Reference & & 440 & $25(5.4)$ & $8.0(5.2,12.2)$ & Reference & \\
\hline Second tertile & 448 & $17(3.7)$ & $4.3(2.4,7.3)$ & $1.00(0.50,1.98)$ & 0.99 & 446 & $19(4.1)$ & $4.4(2.6,7.3)$ & $0.93(0.49,1.72)$ & 0.81 \\
\hline Third tertile & 446 & $19(4.1)$ & $4.2(2.5,6.9)$ & $0.95(0.48,1.89)$ & 0.88 & 431 & $34(7.3)$ & $10.4(7.2,14.6)$ & $1.31(0.74,2.35)$ & 0.36 \\
\hline \multicolumn{11}{|l|}{ HDL-cholesterol } \\
\hline First tertile & 440 & $22(4.8)$ & $5.5(3.4,8.7)$ & Reference & & 431 & $31(6.7)$ & $9.9(6.6,14.3)$ & Reference & \\
\hline Second tertile & 440 & $23(5.0)$ & $6.3(4.0,10.0)$ & $1.15(0.63,2.13)$ & 0.64 & 434 & $28(6.1)$ & $7.5(4.9,11.3)$ & $0.89(0.52,1.50)$ & 0.66 \\
\hline Third tertile & 454 & $8(1.7)$ & $1.8(1.0,3.8)$ & $0.35(0.14,0.81)$ & 0.01 & 444 & $19(4.1)$ & $6.4(4.0,10.0)$ & $0.52(0.27,0.97)$ & 0.04 \\
\hline \multicolumn{11}{|c|}{ Total cholesterol/HDL-cholesterol ratio } \\
\hline First tertile & 450 & $12(2.6)$ & $3.1(1.7,5.6)$ & Reference & & 444 & $18(3.9)$ & $6.0(3.6,9.6)$ & Reference & \\
\hline Second tertile & 444 & $19(4.1)$ & $4.9(2.9,8.2)$ & $1.65(0.80,3.56)$ & 0.18 & 437 & $25(5.4)$ & $6.1(3.9,9.4)$ & $1.73(0.97,3.15)$ & 0.06 \\
\hline Third tertile & 440 & $22(4.8)$ & $5.4(3.4,8.5)$ & $2.11(1.02,4.56)$ & 0.04 & 428 & $35(7.6)$ & $11.5(7.5,16.1)$ & $4.34(1.98,9.22)$ & 0.0004 \\
\hline \multicolumn{11}{|c|}{ Non-HDL-cholesterol } \\
\hline First tertile & 448 & $14(3.0)$ & $3.7(2.2,6.3)$ & Reference & & 441 & $21(4.5)$ & $6.7(4.2,10.4)$ & Reference & \\
\hline Second tertile & 443 & $19(4.1)$ & $4.9(2.9,8.2)$ & $1.33(0.65,2.76)$ & 0.43 & 437 & $25(5.4)$ & $6.2(4.0,9.5)$ & $1.18(0.65,2.18)$ & 0.58 \\
\hline Third tertile & 443 & $20(4.3)$ & $4.6(2.8,7.5)$ & $1.49(0.72,3.16)$ & 0.28 & 431 & $32(6.9)$ & $10.1(6.7,14.3)$ & $1.51(0.83,2.80)$ & 0.18 \\
\hline \multicolumn{11}{|l|}{ LDL-cholesterol } \\
\hline First tertile & 432 & $14(3.1)$ & $4.1(2.4,6.9)$ & Reference & & 426 & $21(4.7)$ & $6.6(4.2,10.4)$ & Reference & \\
\hline Second tertile & 427 & $19(4.3)$ & $5.1(3.0,8.4)$ & $1.37(0.67,2.85)$ & 0.39 & 423 & $24(5.4)$ & $6.7(4.3,10.3)$ & $1.18(0.64,2.19)$ & 0.59 \\
\hline Third tertile & 428 & $19(4.3)$ & $4.7(2.9,7.7)$ & $1.37(0.65,2.92)$ & 0.40 & 417 & $30(6.7)$ & $9.1(6.2,13.1)$ & $1.40(0.76,2.61)$ & 0.28 \\
\hline \multicolumn{11}{|l|}{ Triacylglycerol } \\
\hline First tertile & 447 & $16(3.5)$ & $4.0(2.3,7.0)$ & Reference & & 443 & $20(4.3)$ & $4.7(2.9,7.7)$ & Reference & \\
\hline Second tertile & 445 & $18(3.9)$ & $5.3(3.1,8.8)$ & $1.51(0.76,3.05)$ & 0.24 & 435 & $28(6.1)$ & $9.6(6.0,13.9)$ & $1.41(0.78,2.57)$ & 0.25 \\
\hline Third tertile & 445 & $19(4.1)$ & $4.3(2.6,6.9)$ & $1.49(0.75,2.99)$ & 0.25 & 434 & $30(6.5)$ & $8.9(6.1,12.8)$ & $1.61(0.90,2.94)$ & 0.11 \\
\hline \multicolumn{11}{|l|}{ ApoA-I } \\
\hline First tertile & 441 & $23(5.0)$ & $6.6(4.1,10.4)$ & Reference & & 432 & $32(6.9)$ & $10.3(6.8,15.0)$ & Reference & \\
\hline Second tertile & 443 & $22(4.7)$ & $4.9(3.0,7.7)$ & $1.03(0.56,1.89)$ & 0.93 & 437 & $27(5.8)$ & $8.5(5.6,12.6)$ & $0.86(0.50,1.45)$ & 0.56 \\
\hline Third tertile & 455 & $9(1.9)$ & $2.6(1.3-5.2)$ & $0.43(0.18,0.92)$ & 0.03 & 446 & $19(4.1)$ & $5.2(3.2,8.4)$ & $0.53(0.28,0.96)$ & 0.04 \\
\hline \multicolumn{11}{|l|}{ ApoA-II } \\
\hline First tertile & 441 & $23(5.0)$ & $6.0(3.8,9.5)$ & Reference & & 433 & $31(6.7)$ & $9.7(6.5,14.1)$ & Reference & \\
\hline Second tertile & 447 & $17(3.7)$ & $4.3(2.5,7.3)$ & $0.86(0.44,1.65)$ & 0.65 & 446 & $18(3.9)$ & $6.0(3.6,9.7)$ & $0.60(0.32,1.08)$ & 0.09 \\
\hline Third tertile & 451 & $14(3.0)$ & $3.6(2.0,6.3)$ & $0.81(0.39,1.62)$ & 0.55 & 436 & $29(6.2)$ & $8.1(5.5,11.8)$ & $1.29(0.75,2.22)$ & 0.36 \\
\hline \multicolumn{11}{|l|}{ ApoB-100 } \\
\hline First tertile & 446 & $18(3.9)$ & $4.4(2.7,7.0)$ & Reference & & 436 & $28(6.0)$ & $8.9(5.9,13.2)$ & Reference & \\
\hline Second tertile & 445 & $19(4.1)$ & $5.0(2.9,8.4)$ & $1.32(0.68,2.57)$ & 0.40 & 443 & $21(4.5)$ & $6.0(3.7,9.6)$ & $0.83(0.46,1.48)$ & 0.53 \\
\hline Third tertile & 448 & $17(3.7)$ & $4.3(2.5,7.2)$ & $1.35(0.68,2.67)$ & 0.39 & 436 & $29(6.2)$ & $8.8(6.0,12.6)$ & $1.36(0.78,2.35)$ & 0.27 \\
\hline
\end{tabular}

the incidence of major PAD increased in the upper tertiles of both total cholesterol/HDL-cholesterol ratio and non-HDLcholesterol. These associations were independent on putative confounding variables including key cardiovascular risk factors and were mainly reliable after treating all-cause death and coronary events as competing risks. Comparable results were observed when we considered lower-limb amputation and requirement of revascularisation individually as secondary endpoints.

Few studies have examined the relationship between lipoproteins and PAD in people with type 2 diabetes and results have been contrasting. Reduced HDL-cholesterol 
Table 3 (continued)

\begin{tabular}{|c|c|c|c|c|c|c|c|c|c|c|}
\hline \multirow[t]{2}{*}{ Variable } & \multicolumn{5}{|c|}{ Lower-limb amputation during follow-up ${ }^{a}$} & \multicolumn{5}{|c|}{ Lower-limb arterial revascularisation during follow-up ${ }^{\mathrm{b}}$} \\
\hline & No, $n$ & Yes, $n(\%)$ & $\begin{array}{l}10 \text { year } \\
\text { cumulative } \\
\text { incidence, } \\
\%(95 \% \mathrm{CI})^{\mathrm{c}}\end{array}$ & $\begin{array}{l}\mathrm{HR} \\
(95 \% \mathrm{CI})^{\mathrm{d}}\end{array}$ & $p$ value & No, $n$ & Yes, $n(\%)$ & $\begin{array}{l}10 \text { year } \\
\text { cumulative } \\
\text { incidence, } \\
\%(95 \% \mathrm{CI})^{\mathrm{c}}\end{array}$ & $\begin{array}{l}\text { HR } \\
(95 \% \text { CI })^{d}\end{array}$ & $p$ value \\
\hline \multicolumn{11}{|l|}{$\operatorname{Lp}(a)$} \\
\hline First tertile & 447 & $17(3.7)$ & $4.7(2.8,7.9)$ & Reference & & 448 & $16(3.4)$ & $5.4(3.1,9.2)$ & Reference & \\
\hline Second tertile & 446 & $18(3.9)$ & $4.9(2.9,8.0)$ & $0.77(0.39,1.54)$ & 0.46 & 441 & $23(5.0)$ & $7.1(4.6,10.8)$ & $1.11(0.58,2.16)$ & 0.75 \\
\hline Third tertile & 446 & $19(4.1)$ & $4.1(2.5,6.8)$ & $0.75(0.37,1.52)$ & 0.43 & 426 & $39(8.4)$ & $11.0(7.8,15.2)$ & $1.60(0.90,2.99)$ & 0.11 \\
\hline \multicolumn{11}{|c|}{${ }^{\mathrm{a}}$ Analyses were performed in participants without a baseline history of lower-limb amputation } \\
\hline \multicolumn{11}{|c|}{${ }^{\mathrm{b}}$ Analyses were performed in participants without a baseline history of lower-limb revascularisation } \\
\hline \multicolumn{11}{|c|}{${ }^{\mathrm{c}}$ The 10 year cumulative incidences, with associated $95 \%$ CIs, were estimated using the Kaplan-Meier survival analyses } \\
\hline \multicolumn{11}{|c|}{$\begin{array}{l}{ }^{\mathrm{d}} \mathrm{HRs} \text {, with associated } 95 \% \text { CIs, were computed using Cox proportional hazards regression models, for lower-limb amputation or revascularisation in the } \\
\text { second and third tertiles vs the first tertile (reference) of plasma concentrations of lipoproteins, adjusting for sex, age, duration of diabetes, BMI, systolic } \\
\text { BP, urinary ACR, eGFR, history of tobacco smoking (never, former, current), history of diabetic retinopathy and use of antihypertensive treatment, } \\
\text { statin, metformin and insulin therapy }\end{array}$} \\
\hline \multicolumn{11}{|c|}{$p<0.05$ was significant } \\
\hline
\end{tabular}

concentration was an independent risk factor for PAD in the UK Prospective Diabetes Study (UKPDS) [16]. In contrast, no independent association was observed between lipid variables and PAD in the Action in Diabetes and Vascular Disease: PreterAx and DiamicroN Modified-Release Controlled Evaluation (ADVANCE) and the Bypass Angioplasty Revascularisation Investigation in Type 2 Diabetes (BARI2D) studies [17, 18]. The lipoproteins' profiles (decreased HDL-cholesterol and increased LDL-cholesterol and atherogenic lipids) seem to be more consistent in the general population with PAD than in people with diabetes [19-21]. The definitions of PAD were comparable in studies of diabetic and non-diabetic individuals, varying from abnormal anklebrachial index to symptomatic PAD including intermittent claudication and requirement of revascularisation [16-21]. The principal difference between the two populations could be that amputations and microvascular disease are more common in individuals with diabetes [3, 17], albeit the involvement of microvascular disease in PAD was also reported in individuals without diabetes [22].

Overall, the inverse association between plasma HDLcholesterol and the risk of CVD is among the most consistent and reproducible associations in epidemiological studies [23-25] but whether this association is causal remains unclear. The most recognised function of HDL lipoproteins is reverse cholesterol transport, leading to the removal of excess cholesterol from peripheral tissues to the liver. The uptake and transport of cholesterol by HDL for hepatic excretion prevents and potentially reverses its peripheral accumulation in arteries [26-28]. HDL particles have been also associated with some other valuable effects, including antioxidative, antithrombotic, anti-inflammatory and vasodilatory functions [29]. Normalisation of HDL-cholesterol has been described as an unmet need in the management of patients with high cardiovascular risk, including type 2 diabetes [30], but Mendelian randomisation studies have generated scepticism about the hypothetical HDL causality [31, 32]. Furthermore, randomised clinical trials showed that drugs increasing plasma HDL-cholesterol did not reduce the risk of atherosclerotic CVD. Cholesteryl ester transfer protein (CETP) inhibitors have either had negative, neutral or only minor beneficial effects on cardiovascular outcomes despite substantial increase in HDL-cholesterol levels [33-36]. The paradoxical inability of HDL-cholesterol-raising therapies to reduce cardiovascular adverse events may be explained by a highly complex and multifunctional biology of the HDL lipoprotein system. A recent study has shown significant associations between dysfunctional HDL particles and increased risk of acute coronary syndrome and its manifestations in individuals at high cardiovascular risk [37]. Treatments targeting HDL functions could be a potential therapeutic approach. Meanwhile, HDL-cholesterol measurement remains a key component of CVD risk stratification and is still recommended as such [38].

As far as we know, this is the first investigation of the relationship between plasma concentrations of apolipoproteins and the risk of major PAD in a prospective cohort of people with type 2 diabetes. Class A apolipoproteins are the major structural and functional protein components of HDL; they stabilise HDL lipoprotein structure, solubilise their lipid component and help in reverse cholesterol transport. They also act as ligands for cellular receptor binding and enzyme 
activators or inhibitors. ApoA-I accounts for approximately $70 \%$ of HDL structure while ApoA-II corresponds to about $20 \%$. Our findings pointed out an independent and reliable association between high ApoA-I concentrations and reduced risk of major PAD. Plasma concentrations of ApoA-II were also inversely associated with a greater prevalence of PAD at baseline and increased risk of incident PAD during follow-up but the latter association was not consistent and was mainly dependent on confounding variables. We did not observe evidence for significant interaction between HLDcholesterol and ApoA-I in their association with the risk of major PAD, suggesting that these lipid variables may interact differently on this condition, although our data cannot allow any mechanistic conclusion. ApoA-I has also been linked to CVD [25, 37]; a large meta-analysis emphasised not only an inverse association between ApoA-I and a reduction of major cardiovascular events but also showed that increase in ApoA-I concentrations led to decreased cardiovascular risk among statin-treated patients [25].

We did not observe significant association between triacylglycerol, ApoB-100 or Lp(a) and major PAD. Also, plasma concentrations of LDL-cholesterol were not significantly associated with increased risk of major PAD during follow-up $(p=$ $0.05)$. The fact that LDL-cholesterol was not measured in our cohort but was only estimated using the Friedewald formula after excluding participants with high levels of triacylglycerol may have mitigated our results. Nevertheless, our findings are consistent with those of a recent prospective study reporting a significant association between low standard plasma concentration of HDL-cholesterol (but not LDL-cholesterol) and incident PAD events among women without known CVD at baseline [21]. However, this study showed strong associations between excess incident PAD and a series of atherogenic lipidomic features: reduced HDL; and elevated LDL particles, small LDL particles and medium and very large VLDL particles. Of note, we have observed an increased risk of incident major $\mathrm{PAD}$ in the top tertile of non-HDL-cholesterol, which estimates total concentrations of all atherogenic ApoB-containing lipoproteins including triacylglycerol-rich particles in VLDLs and their remnants. This association was independent of confounders without evidence for significant interaction with HDL-cholesterol, ApoA-I or use of statins. However, this association did not persist after treating all-cause death or coronary events as competing risks, although having a similar magnitude to the association observed in the primary analyses. In addition, plasma concentrations of non-HDL-cholesterol was not associated with prevalent PAD. This difference cannot be explained by the baseline characteristics of participants in the prevalent and the incident PAD groups, as these were roughly comparable. A potential explanation for this difference is that incident PAD seemed to be more likely related to large-vessel disease than prevalent PAD. Indeed, the requirement of revascularisation accounted for $72 \%$ of incident PAD while limb loss was more frequent at baseline $(5.0 \%$ vs $3.9 \%$ at the time of endpoint), including mainly minor amputation (74\% at baseline vs $45 \%$ at the time of endpoint). Additionally, arterial stenosis with significant haemodynamic effects was observed in $95 \%$ of amputees at the time of endpoint (vs 59\% at baseline). Taken together, these findings suggest that increased nonHDL-cholesterol could mainly reflect a high risk of macrovascular disease in patients with PAD. Non-HDL-C has been suggested as a pragmatic and cost-effective cardiovascular biomarker, especially in people with type 2 diabetes [38, 39].

Our study also highlights the total cholesterol/HDLcholesterol ratio as a strong and consistent lipid biomarker for the risk of major PAD. A high total cholesterol/HDLcholesterol ratio was associated with both prevalent and incident PAD. This association was independent of relevant confounders, persisted when we dealt with all-cause death or coronary events as competing risk, and was also reliable when we considered incident lower-limb amputation and revascularisation individually. These findings are consistent with those of an earlier study reporting total cholesterol/ HDL-cholesterol ratio as a strong and independent predictor of PAD in a nested case-control cohort from the Physicians' Health Study [20].

The key strength of our work is the investigation of a prospective cohort of individuals with type 2 diabetes collecting a wide range of clinical and biological features at baseline with adjudicated outcomes during a median followup of 7 years. We have measured a set of lipid and apolipoprotein compounds, which may reflect at least partly a factual picture of lipoproteins' profile in individuals with type 2 diabetes. However, our study may not be representative of all populations with type 2 diabetes as SURDIAGENE is a French mono-centre inpatient cohort. Also, 45\% participants were on statin therapy at baseline, which may influence our findings as this treatment reduces the risk of PAD events [40]. However, all our analyses were adjusted for statin use and we did not observe significant interaction between statin use and relevant lipids biomarkers in their relationship with major PAD. On the other hand, we assessed only baseline use of statin, leaving some uncertainty about potential increase in statin use during follow-up that may possibly bias our results. The issue is that we cannot assess time-varying hazards as we do not have data regarding the use of statins over time. Our investigation may also omit potential association between lipid variables and early stages of PAD as we have evaluated only advanced PAD-related events. Finally, we did not have accurate and comprehensive data regarding peripheral neuropathy. Of note, all amputees had a strong evidence of PAD (abolition of peripheral pulses, intermittent claudication or lower-limb artery stenosis with haemodynamic effects). At the same time, peripheral diabetic neuropathy and foot infection were reported in $51-76 \%$ of amputees, supporting the notion that lower-limb amputation is a dramatic consequence 
of several concomitant complications including microvascular, macrovascular and infectious disease.

In conclusion, we have observed independent and reliable associations between plasma concentrations of HDL-cholesterol, total cholesterol/HDL-cholesterol ratio and ApoA-I and the prevalence at baseline and the incidence during follow-up of major PAD in individuals with type 2 diabetes. Increased non-HDL-cholesterol concentrations were also associated with increased incidence of major PAD. Our findings may help to identify a specific lipoprotein's profile in individuals with type 2 diabetes at high risk of major PAD.

Supplementary Information The online version contains peer-reviewed but unedited supplementary material available at https://doi.org/10.1007/ s00125-020-05326-x.

Acknowledgements We thank all the participants in the SURDIAGENE cohort and E. Migault (Inserm CIC1402, Poitiers, France). The staff of the Diabetes Department in Poitiers university hospital are acknowledged for their help with data collection and monitoring. We thank S. Brishoual (Biological Resources Center, BRC BB-0033-00068, Poitiers, France) for biological determinations and also thank A. Pavy and M.-C. Pasquier (Information Technology Department, CHU de Poitiers, Poitiers, France). We thank Y. Mohammedi (Saint-Genès La Salle high school, Bordeaux, France) for his help in preparing the graphical abstract.

Data availability The datasets generated and analysed during the current study are available from the corresponding author on reasonable request.

Funding The SURDIAGENE cohort was supported by grants from the French Ministry of Health (PHRC-Poitiers 2004; PHRC-IR 2008), the Association Française des Diabétiques (Research Grant 2003) and the Groupement pour l'Etude des Maladies Métaboliques et Systémiques (GEMMS Poitiers, France). The apolipoprotein assessments were supported by a grant received by MC from 'Fondation de France' (CARDIO No. 00086491). We are also grateful to the Biogenouest CORSAIRE core facility for financial support.

Authors' relationships and activities LP reports personal fees and nonfinancial support from Sanofi, Novo Nordisk, Eli Lilly and MSD. OB reports personal fees and non-financial support from MSD and Sanofi. MM reports personal fees and non-financial support from Novo Nordisk, Merck, Eli Lilly and Servier. SH reports personal fees and grants from Bayer, Merck/MSD Chibret, Mundipharma, NovoNordisk, Valbiotis, Astra Zeneca, Boehringer, Eli Lilly, Novartis, Sanofi and Servier. The other authors declare that there are no relationships or activities that might bias, or be perceived to bias, their work.

Contribution statement $\mathrm{CB}$, PJS, SH and KM designed the study and researched data. $\mathrm{CB}$ and $\mathrm{KM}$ drafted the manuscript. PJS and SH contributed to discussion and reviewed/edited the manuscript. MC and VB researched data, contributed to discussion and reviewed/edited the manuscript. LP, EG, SR, FS, OB, LBB, GV, MM, RR and VR participated in the analyses and the interpretation of data and contributed to discussion and reviewed/edited the manuscript. All authors approved the current version of the manuscript. KM is the guarantor of this work and, as such, had full access to all the data in the study and take responsibility for the integrity of the data and the accuracy of the data analyses.

\section{References}

1. Criqui MH, Aboyans V (2015) Epidemiology of peripheral artery disease. Circ Res 116:1509-1526. https://doi.org/10.1161/ CIRCRESAHA.116.303849

2. Nativel M, Potier L, Alexandre L et al (2018) Lower extremity arterial disease in patients with diabetes: a contemporary narrative review. Cardiovasc Diabetol 17:138

3. Jude EB, Oyibo SO, Chalmers N, Boulton AJ (2001) Peripheral arterial disease in diabetic and nondiabetic patients: a comparison of severity and outcome. Diabetes Care 24:1433-1437. https://doi. org/10.2337/diacare.24.8.1433

4. Fowkes FG, Rudan D, Rudan I et al (2013) Comparison of global estimates of prevalence and risk factors for peripheral artery disease in 2000 and 2010: a systematic review and analysis. Lancet 382: 1329-1340. https://doi.org/10.1016/S0140-6736(13)61249-0

5. Sampson UK, Fowkes FG, McDermott MM et al (2014) Global and regional burden of death and disability from peripheral artery disease: 21 world regions, 1990 to 2010. Glob Heart 9:145-158 e121

6. Fosse S, Hartemann-Heurtier A, Jacqueminet S, Ha Van G, Grimaldi A, Fagot-Campagna A (2009) Incidence and characteristics of lower limb amputations in people with diabetes. Diabet Med 26:391-396. https://doi.org/10.1111/j.1464-5491.2009.02698.x

7. Mueller T, Hinterreiter F, Luft C, Poelz W, Haltmayer M, Dieplinger B (2014) Mortality rates and mortality predictors in patients with symptomatic peripheral artery disease stratified according to age and diabetes. J Vasc Surg 59:1291-1299. https://doi.org/10.1016/j.jvs.2013.11.063

8. Mohammedi K, Woodward M, Hirakawa Y et al (2016) Presentations of major peripheral arterial disease and risk of major outcomes in patients with type 2 diabetes: results from the ADVANCE-ON study. Cardiovasc Diabetol 15:129

9. Mooradian AD (2009) Dyslipidemia in type 2 diabetes mellitus. Nat Clin Pract Endocrinol Metab 5:150-159. https://doi.org/10. 1038/ncpendmet1066

10. Michos ED, McEvoy JW, Blumenthal RS (2019) Lipid Management for the Prevention of Atherosclerotic Cardiovascular Disease. N Engl J Med 381:1557-1567. https:// doi.org/10.1056/NEJMra1806939

11. Hackam DG, Hegele RA (2019) Cholesterol Lowering and Prevention of Stroke. Stroke 50:537-541. https://doi.org/10.1161/ STROKEAHA.118.023167

12. Saulnier PJ, Gand E, Velho G et al (2017) Association of Circulating Biomarkers (Adrenomedullin, TNFR1, and NTproBNP) With Renal Function Decline in Patients With Type 2 Diabetes: A French Prospective Cohort. Diabetes Care 40:367374. https://doi.org/10.2337/dc16-1571

13. Croyal M, Kaabia Z, Leon L et al (2018) Fenofibrate decreases plasma ceramide in type 2 diabetes patients: A novel marker of CVD? Diabetes Metab 44:143-149. https://doi.org/10.1016/j. diabet.2017.04.003

14. Croyal M, Ouguerram K, Passard M et al (2015) Effects of Extended-Release Nicotinic Acid on Apolipoprotein (a) Kinetics in Hypertriglyceridemic Patients. Arterioscler Thromb Vasc Biol 35:2042-2047. https://doi.org/10.1161/ATVBAHA.115.305835

15. Langsted A, Kamstrup PR, Nordestgaard BG (2019) High lipoprotein(a) and high risk of mortality. Eur Heart J 40:2760 2770. https://doi.org/10.1093/eurheartj/ehy902 
16. Adler AI, Stevens RJ, Neil A, Stratton IM, Boulton AJ, Holman RR (2002) UKPDS 59: hyperglycemia and other potentially modifiable risk factors for peripheral vascular disease in type 2 diabetes. Diabetes Care 25:894-899. https://doi.org/10.2337/diacare.25.5. 894

17. Mohammedi K, Woodward M, Hirakawa Y et al (2016) Microvascular and Macrovascular Disease and Risk for Major Peripheral Arterial Disease in Patients With Type 2 Diabetes. Diabetes Care 39:1796-1803. https://doi.org/10.2337/dc16-0588

18. Althouse AD, Abbott JD, Forker AD et al (2014) Risk factors for incident peripheral arterial disease in type 2 diabetes: results from the Bypass Angioplasty Revascularization Investigation in type 2 Diabetes (BARI 2D) Trial. Diabetes Care 37:1346-1352. https:// doi.org/10.2337/dc13-2303

19. Kennedy M, Solomon C, Manolio TA et al (2005) Risk factors for declining ankle-brachial index in men and women 65 years or older: the Cardiovascular Health Study. Arch Intern Med 165:1896-1902. https://doi.org/10.1001/archinte.165.16.1896

20. Ridker PM, Stampfer MJ, Rifai N (2001) Novel risk factors for systemic atherosclerosis: a comparison of C-reactive protein, fibrinogen, homocysteine, lipoprotein(a), and standard cholesterol screening as predictors of peripheral arterial disease. JAMA 285: 2481-2485. https://doi.org/10.1001/jama.285.19.2481

21. Aday AW, Lawler PR, Cook NR, Ridker PM, Mora S, Pradhan AD (2018) Lipoprotein Particle Profiles, Standard Lipids, and Peripheral Artery Disease Incidence. Circulation 138:2330-2341. https://doi.org/10.1161/CIRCULATIONAHA.118.035432

22. Beckman JA, Duncan MS, Damrauer SM et al (2019) Microvascular Disease, Peripheral Artery Disease, and Amputation. Circulation 140:449-458. https://doi.org/10.1161/ CIRCULATIONAHA.119.040672

23. Di Angelantonio E, Gao P, Pennells L et al (2012) Lipid-related markers and cardiovascular disease prediction. JAMA 307:2499 2506. https://doi.org/10.1001/jama.2012.6571

24. Bartlett J, Predazzi IM, Williams SM et al (2016) Is Isolated Low High-Density Lipoprotein Cholesterol a Cardiovascular Disease Risk Factor? New Insights From the Framingham Offspring Study. Circ Cardiovasc Qual Outcomes 9:206-212. https://doi. org/10.1161/CIRCOUTCOMES.115.002436

25. Boekholdt SM, Arsenault BJ, Hovingh GK et al (2013) Levels and changes of HDL cholesterol and apolipoprotein A-I in relation to risk of cardiovascular events among statin-treated patients: a metaanalysis. Circulation 128:1504-1512. https://doi.org/10.1161/ CIRCULATIONAHA.113.002670

26. Khera AV, Cuchel M, de la Llera-Moya M et al (2011) Cholesterol efflux capacity, high-density lipoprotein function, and atherosclerosis. N Engl J Med 364:127-135. https://doi.org/10.1056/ NEJMoa1001689

27. Saleheen D, Scott R, Javad S et al (2015) Association of HDL cholesterol efflux capacity with incident coronary heart disease events: a prospective case-control study. Lancet Diabetes Endocrinol 3:507-513. https://doi.org/10.1016/S2213-8587(15) 00126-6
28. Koekemoer AL, Codd V, Masca NGD et al (2017) Large-Scale Analysis of Determinants, Stability, and Heritability of HighDensity Lipoprotein Cholesterol Efflux Capacity. Arterioscler Thromb Vasc Biol 37:1956-1962. https://doi.org/10.1161/ ATVBAHA.117.309201

29. Rosenson RS, Brewer HB Jr, Ansell BJ et al (2016) Dysfunctional HDL and atherosclerotic cardiovascular disease. Nat Rev Cardiol 13:48-60. https://doi.org/10.1038/nrcardio.2015.124

30. Grant RW, Meigs JB (2007) Prevalence and treatment of low HDL cholesterol among primary care patients with type 2 diabetes: an unmet challenge for cardiovascular risk reduction. Diabetes Care 30:479-484. https://doi.org/10.2337/dc06-1961

31. Voight BF, Peloso GM, Orho-Melander M et al (2012) Plasma HDL cholesterol and risk of myocardial infarction: a mendelian randomisation study. Lancet 380:572-580. https://doi.org/10. 1016/S0140-6736(12)60312-2

32. Holmes MV, Asselbergs FW, Palmer TM et al (2015) Mendelian randomization of blood lipids for coronary heart disease. Eur Heart J 36:539-550. https://doi.org/10.1093/eurheartj/eht571

33. Barter PJ, Caulfield M, Eriksson M et al (2007) Effects of torcetrapib in patients at high risk for coronary events. N Engl $\mathrm{J}$ Med 357:2109-2122. https://doi.org/10.1056/NEJMoa0706628

34. Schwartz GG, Olsson AG, Abt M et al (2012) Effects of dalcetrapib in patients with a recent acute coronary syndrome. N Engl J Med 367:2089-2099. https://doi.org/10.1056/NEJMoa1206797

35. Lincoff AM, Nicholls SJ, Riesmeyer JS et al (2017) Evacetrapib and Cardiovascular Outcomes in High-Risk Vascular Disease. N Engl J Med 376:1933-1942

36. Bowman L, Hopewell JC, Chen F et al (2017) Effects of Anacetrapib in Patients with Atherosclerotic Vascular Disease. N Engl J Med 377:1217-1227. https://doi.org/10.1056/ NEJMoa1706444

37. Soria-Florido MT, Castaner O, Lassale C et al (2020) Dysfunctional High-Density Lipoproteins Are Associated With a Greater Incidence of Acute Coronary Syndrome in a Population at High Cardiovascular Risk: A Nested Case-Control Study. Circulation 141:444-453. https://doi.org/10.1161/CIRCULATIONAHA.119. 041658

38. Mach F, Baigent C, Catapano AL et al (2020) 2019 ESC/EAS Guidelines for the management of dyslipidaemias: lipid modification to reduce cardiovascular risk. Eur Heart J 41:111-188. https:// doi.org/10.1093/eurheartj/ehz455

39. Welsh C, Celis-Morales CA, Brown R et al (2019) Comparison of Conventional Lipoprotein Tests and Apolipoproteins in the Prediction of Cardiovascular Disease. Circulation 140:542-552. https://doi.org/10.1161/CIRCULATIONAHA.119.041149

40. Arya S, Khakharia A, Binney ZO et al (2018) Association of Statin Dose With Amputation and Survival in Patients With Peripheral Artery Disease. Circulation 137:1435-1446. https://doi.org/10. 1161/CIRCULATIONAHA.117.032361

Publisher's note Springer Nature remains neutral with regard to jurisdictional claims in published maps and institutional affiliations. 